\title{
Giovanni Battista Monteggia (1762-1815): el cirujano detrás del epónimo
}

\author{
Giovanni Battista Monteggia (1762-1815): the surgeon behind the eponymous
}

\section{Leopoldo Mario Aguayo González*}

${ }^{*}$ Catedrático e Investigador en Historia de la Medicina, Universidad Latina de México, Celaya, Guanajuato. Profesor de Músculo Esquelético en la misma Universidad. Profesor de Biomecánica, Prótesis y Órtesis, Ingeniería Biomédica, Universidad de Celaya. Profesor e Investigador en Historia de la Ortopedia y Traumatología. Federación Mexicana de Colegios de Ortopedia y Traumatología AC (FEMECOT). Sociedad Latinoamericana de Ortopedia y Traumatología Infantil (SLAOTI). Académico Sociedad Potosina de Estudios Médicos AC (SPEM). Titular del Comité de Historia y Arte de la (FEMECOT), Mesa Directiva 2020-2022.

\section{Resumen}

Giovanni Battista Monteggia nació sólo 19 años después de que el cirujano francés Francois Gigot de la Peyronie (1678-1747) consiguiera la separación definitiva entre barberos y cirujanos por ordenanza real de Luis XV el 23 de abril de 1743. Muere en la primera veintena del siglo XIX, que vio nacer en el año de 1846 el advenimiento de la anestesia y de la antisepsia en 1867. Monteggia, ilustre cirujano y prolífico escritor médico, desarrolla toda su carrera en Milán, donde muere a la edad de 52 años; fue miembro del Instituto de la Unidad Italiana, cirujano principal del Hospital Mayor de Milán, socio de la Academia Italiana, de la de Génova, Mantua, Venecia, Livorno, Florencia y Lucca. Monteggia vivía cerca del Ospedale Maggiore di Milano, donde por muchos años se dedicó al cuidado de los enfermos y a la formación de sus discípulos. Dos años antes de su muerte ve la luz, la última edición de su obra médica monumental en ocho volúmenes titulada Instituzioni Chirurgiche, editada en 1813 por Giuseppe Maspero de Milán. Monteggia fue uno de los pioneros en el análisis del cuadro clínico de la parálisis infantil o poliomielitis descrita por primera vez en 1784 por Michael Underwood (1736-1820). Y en la cúspide de su carrera fue el encargado de controlar la aplicación de la vacunación contra la viruela en Milán, desarrollada inicialmente por Edward Anthony Jenner (1749-1823) el 14 de mayo de 1798 en el Reino Unido. En el mes de abril de 1816, a poco más de un año y tres meses después de su muerte acaecida el 17 de enero de 1815, le fue erigido un busto de mármol colocado en el Hospital Mayor de Milán.

Palabras clave: Historia de la medicina, historia de la traumatología y de las fracturas-luxaciones.

\begin{abstract}
Monteggia was born only 19 years after the French surgeon; Francois Gigot de la Peyronie (1678-1747) gets the final separation between barbers and surgeons by royal ordinance of Louis XV on April 23, 1743. He dies in the first two decades of the nineteenth century, who saw the advent in the year of 1846, of anesthesia, and antisepsis in 1867. Monteggia distinguished surgeon and prolific medical writer, develops his entire career in Milan, where he died at the age of 52 years; he was Member of the Institute of the United Italian, chief of surgeons of the Ospedale Maggiore of Milan, a partner at the Italian Academy, of Genoa, Mantua, Venice, Livorno, Florence and Lucca. Monteggia lived near the hospital where for many years was devoted to patient care and training of his disciples. Two years before his death saw the light, the last edition of his monumental medical work in eight volumes entitled Instituzioni Chirurgiche published in 1813 by Giuseppe Maspero of Milan, Monteggia was among one of the pioneers in the clinical analysis of infantile paralysis or polio, described per prima in 1784 by Michael Underwood (1736-1820). And at the cuspid of his career was responsible for monitoring the implementation of vaccination against smallpox in Milan, initially developed by Edward Anthony Jenner (1749-1823) on May 14, 1798 in the United Kingdom. On April 1816, one year and three months after his death, on January 17th 1815, was erected a marble bust located in the Ospedale Maggiore of Milan.
\end{abstract}

Keywords: History of medicine, history of traumatology and fractures-dislocations.

Correspondencia:

Dr. Leopoldo Mario Aguayo González

E-mail: aguayoleopoldo@gmail.com

Recibido: 06-02-2021. Aceptado: 07-02-2021.

Citar como: Aguayo GLM. Giovanni Battista Monteggia (1762-1815): el cirujano detrás del epónimo. Orthotips. 2021; 17 (1): 59-63. https:// dx.doi.org/10.35366/99169 


\section{Introducción}

Giovanni Battista Monteggia nació sólo 19 años después de que el cirujano francés Francois Gigot de la Peyronie (1678-1747) consiguiera la separación definitiva entre barberos y cirujanos por ordenanza real de Luis XV el 23 de abril de 1743. Muere en la primera veintena del siglo XIX, que vio nacer en el año de 1846 el advenimiento de la anestesia y de la antisepsia en 1867 (Figura 1).

Monteggia, ilustre cirujano y prolífico escritor médico, desarrolla toda su carrera en Milán, donde muere a la edad de 52 años; fue miembro del Instituto de la Unidad Italiana, cirujano principal del Hospital Mayor de Milán, socio de la Academia Italiana, de la de Génova, Mantua, Venecia, Livorno, Florencia y Lucca. Monteggia vivía cerca del Ospedale Maggiore di Milano, donde por muchos años se dedicó al cuidado de los enfermos y a la formación de sus discípulos.

Dos años antes de su muerte ve la luz, la última edición de su obra médica monumental en ocho volúmenes titulada Instituzioni Chirurgiche, editada en 1813 por Giuseppe Maspero de Milán. Monteggia fue uno de los pioneros en el análisis del cuadro clínico de la parálisis infantil o poliomielitis descrita por primera vez en 1784 por Michael Underwood (1736-1820).

Y en la cúspide de su carrera fue el encargado de controlar la aplicación de la vacunación contra la viruela en Milán, desarrollada inicialmente por Edward Anthony Jenner (1749-1823) el 14 de mayo de 1798 en el Reino Unido. En el mes de abril de 1816, a poco más de un año y tres meses después de su muerte acaecida el 17 de enero de 1815, le fue erigido un busto de mármol colocado en el Hospital Mayor de Milán.

\section{La vida de G. B. Monteggia}

Giovanni Battista Monteggia nace el 08 de agosto de 1762 en el puerto de Laverno de la provincia de Varese, en la rivera del lago Como, región de Lombardía, Italia; sus padres fueron Gian Antonio Monteggia y Maria Anna Vegezzi, crece en el pequeño villorio de Monteggia en la misma provincia de Varese de donde tomó el apellido la familia de su padre..$^{1-3}$

Su papá trabajaba en la construcción de caminos y obras hidráulicas, envió al joven Giovanni Battista a estudiar en la vecina Pallenza, donde aprendió los rudimentos de la lengua latina; sin embargo, estos estudios fueron de corta duración y de poco provecho. ${ }^{1-3}$

A la edad de 17 años, interesado en dedicarse a la cirugía, llega a la ciudad de Milán donde el 14 de septiembre de 1779 ingresa como alumno interno del Hospital Mayor, recibiendo alojamiento y comida. Téngase presente que en aquel entonces los ayudantes de cirugía iniciaban como simples flebotomianos y enfermeros. ${ }^{1-3}$

Cultiva el estudio de la anatomía con mucho ardor y pronto fue capaz de adquirir tal destreza que sobrepasó a sus compañeros, algunos de ellos satisfechos sólo con la demostración académica. Acude a las lecciones de cirugía impartidas por Pietro Moscati y G. B. Palletta, y tan grande era su deseo de aprender, que no perdía tiempo, llevaba incluso sus libros a la mesa del comedor y con frecuencia pasaba las horas de la noche dedicadas también al estudio. ${ }^{3}$

Por motivos económicos no pudo realizar estudios preparatorios, pero adquiere una sólida cultura teórica de la lectura de los escritos prestados por sus compañeros con mayores recursos financieros. ${ }^{3} \mathrm{G}$. B. Monteggia, perfectamente preparado y a la corta edad de veinte años, se presentó al examen práctico

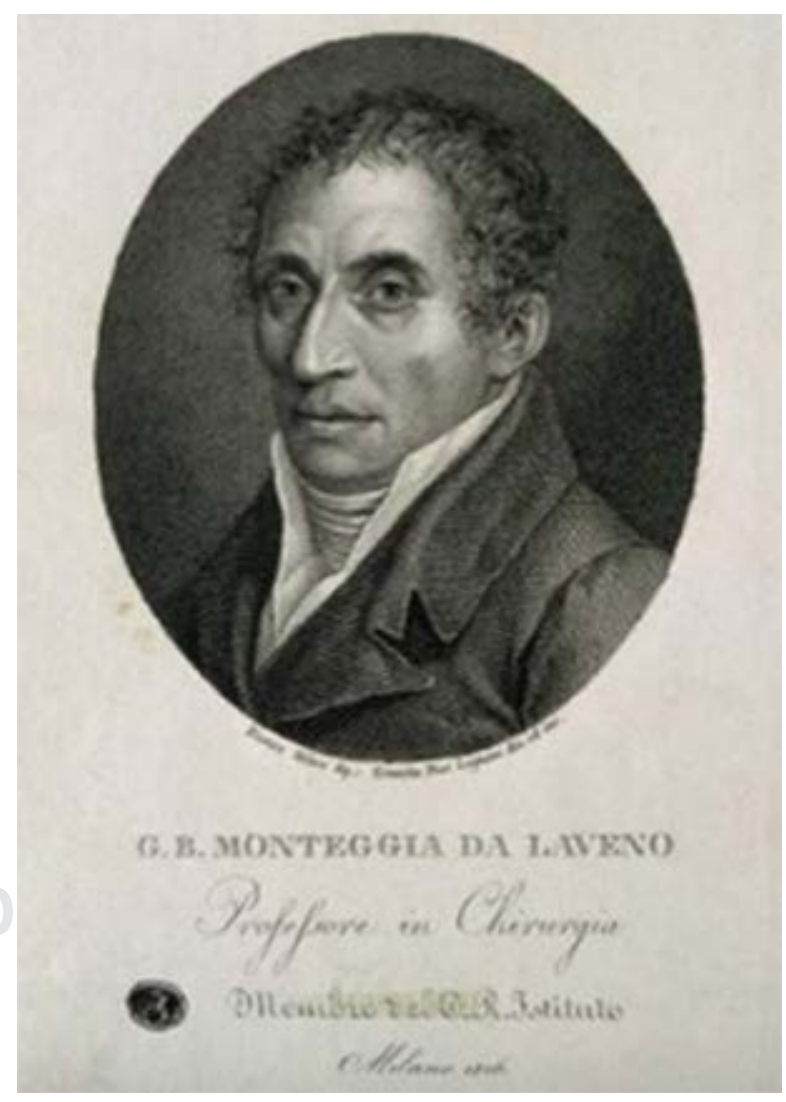

Figura 1: Giovanni Battista Monteggia (1762-1815): el cirujano detrás del epónimo.

Crédito Wellcome Images, Londres. 
de cirugía mayor, en la Universidad de Pavía el día 11 de junio de 1781, superando brillantemente el examen con una especial mención honorífica. ${ }^{1-3}$

En aquella época, el diploma de cirujano tenía un valor netamente inferior al del graduado en medicina; sin embargo, Monteggia, consigue también este diploma tres años después, su conocimiento de la materia quirúrgica era tan grande que obtiene la dispensa de la formalidad establecida por el trienio de la República Cisalpina establecida durante la ocupación napoleónica. ${ }^{3}$

Dotado de excepcional espíritu de observación, anotaba diariamente todos los cambios que se presentaban en el lecho del enfermo, desde los más simples hasta los más raros y difíciles, se instruía continuamente en el arte médico, uniendo la lectura asidua de los libros científicos con la observación práctica diaria. ${ }^{1-3} \mathrm{Y}$ para poder estar siempre bien informado del avance de la cirugía europea de su tiempo, estudia lenguas extranjeras (francés, alemán e inglés) y establece también correspondencia con los principales cirujanos de esos países. ${ }^{3}$

Fue contemporáneo de Pierre Joseph Desault (1744-1795), Francois Chopart (1743-1795), Giovanni Battista Palletta (1747-1830), Antonio Scarpa (17521832), Pietro Moscati (1739-1824) y Enrico Acervi (1785-1827) su primer biógrafo en 1816. ${ }^{1-3}$

Escribe en el año de 1786, en latín, Osservazioni anatomiche patologiche; una revista editada en la Venecia de aquella época la llena de encomios y señalaba «tan interesante es, que una vez que cae en tus manos, es imposible no leerla desde el principio hasta el final con atención ininterrumpida y verdadero placer», fueron traducidas después al italiano y dedicadas a sus ilustres profesores Palletta y Moscati. ${ }^{1-3}$

En el año de 1789 publicó también en latín Fasciculi Pathologici, primera edición, obra en la cual describe las enfermedades simétricas y asimétricas, las úlceras de los miembros inferiores, de los síntomas producidos por las enfermedades cerebrales, de la glándula tiroides, del manejo de las hernias y de otras afecciones morbosas.

Al año siguiente fue nombrado ayudante quirúrgico y un año después profesor de anatomía del Hospital Mayor de Milán con derecho a sueldo y alojamiento. Para poder cumplir mejor sus actividades solicita a la administración del hospital le construyan una habitación junto a la sala de disecciones, pero le fue negada por motivos económicos del propio hospital; sin embargo, la habitación sí fue construida a expensas del párroco Carlo Maria Taverna de la Chiesa de

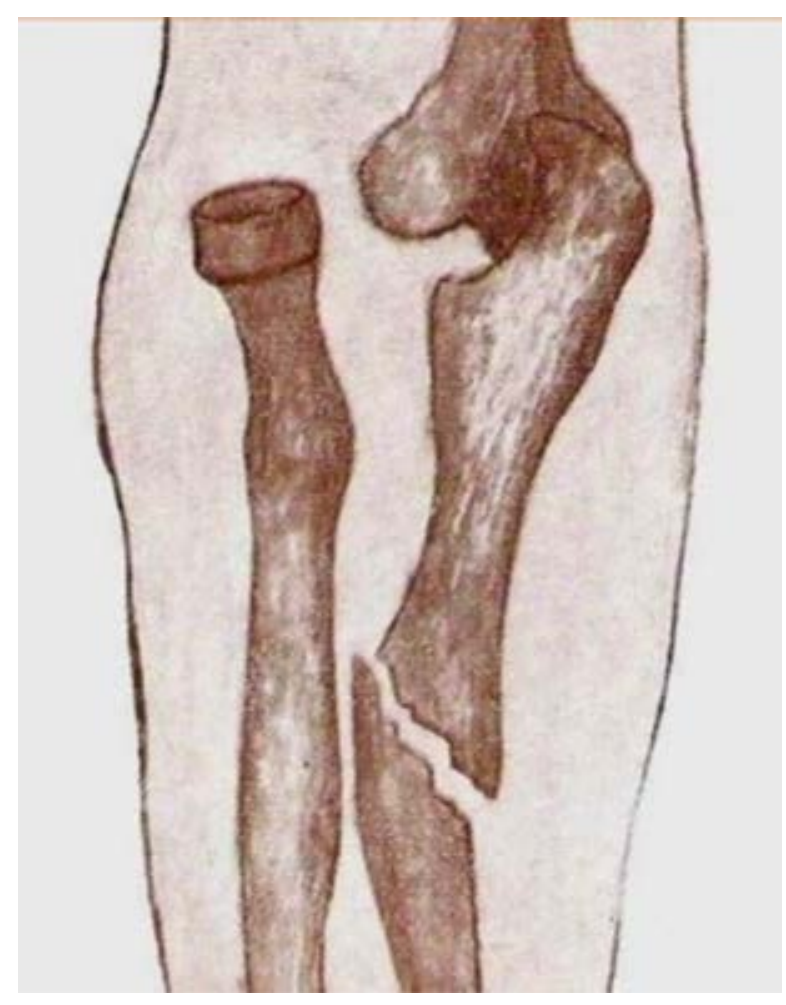

Figura 2: Lesión Monteggia, dibujo del propio autor 1814.

San Nazaro Maggiore, y como reconocimiento a este sacerdote y a su ilustre preceptor Palletta dedica a ambos la segunda edición de Fasciculi Pathologici.

En el año de 1791 fue electo por decreto del Real Tribunal de Apelaciones, primer cirujano de la cárcel y del foro criminal, dependientes del supremo Tribunal de Justicia. Todavía en el mismo año de 1791, publicó traducida del alemán la obra de Johann Friedrich Fritze (1747-1819) Compendio sobre las Enfermedades Venéreas.

Varios años después escribe su propio libro Annotazione pratiche sopra i male veneri, Edit. Giuseppe Galeazzi, Milano 1794, dedicadas a su maestro Pietro Moscati. ${ }^{4}$ En la introducción de este libro consigna el uso de la zarzaparrilla (planta originaria de América y exportada a Europa siglo XVI) para el tratamiento de las enfermedades venéreas; la terapéutica utilizada en aquella época para estas enfermedades era el uso de compuestos mercuriales. También escribe en el mismo libro sobre la artritis gonocócica, ya descrita en 1770 por el austriaco naturalizado francés Francois Xavier Swediaur (1748-1824). ${ }^{3,4}$ Añade también en este libro que durante la autopsia de una mujer muerta de sífilis, accidentalmente se produjo una herida con el cuchillo 
en uno de sus dedos, con lo cual adquiere un grave absceso en la mano que puso en peligro su vida y que fue curado gracias a la habilidad de G. B. Palletta, a quien debió su sanación. ${ }^{4}$ Téngase en cuenta que el uso de los guantes para los procedimientos quirúrgicos y disecciones fue introducido hasta el año de 1890 por William Steward Halsted (1852-1922) del John Hopkins Hospital, EUA, y que la aplicación de las sulfonamidas para el manejo de las infecciones bacterianas sistémicas sólo fue posible hasta la primera mitad del siglo XX, específicamente en 1935 por Gerhard Domagk (1895-1964) de Wuppertal, Alemania.

En el año de 1792, por auto de la Congregación del Hospital Mayor, le fue encargada la misión de dar gratuitamente las lecciones teórico-prácticas a los jóvenes cirujanos en formación. ${ }^{3}$ Publica durante este periodo dos memorias: la primera sobre las fracturas simples de las costillas en la que demuestra como éstas pueden consolidar sin obligar al paciente a guardar cama y la segunda sobre un caso particular de falsa demencia y el uso del opio en su tratamiento. ${ }^{1-3}$

También en 1796 traduce del alemán a su idioma natal El Arte de la Obstetricia de Georg Wilhelm Stein el joven (1773-1870), añadiendo observaciones de su propia y amplia experiencia de cómo se puede conservar la vida del niño durante la gestación y el nacimiento, consigna también la invención del cefalotribo para la extracción del feto muerto; sin embargo, este instrumento, ampliamente demostrado y divulgado en Italia y Francia, fue olvidado por sus contemporáneos y el mérito del invento recae en Jean Louis Baudelocque (1745-1810), quien lo presentó a la Academia de Ciencias de París. ${ }^{1-3}$

El mismo año, a la edad de 32 , se casa con la señorita Giovanna Cremona de una notable familia de Novara, con quien procrea cinco hijos, de los cuales sólo sobreviven tres: una mujer, Francesca, y dos hombres, Luigi y Tito, ambos doctores en derecho. ${ }^{1-3}$

Por otra parte, la exitosa cirugía realizada a Francesco Melzi conde de D'Eril, vicepresidente de la República Cisalpina, a quien habían desahuciado otros médicos dentro y fuera de Italia, le crea mayor fama y fortuna y un insustituible mecenazgo. Gracias a la amistad de este personaje se crea la Facultad de Cirugía del Hospital Mayor que inició su labor en 1800 , el discurso inaugural del 13 de mayo fue del propio Monteggia. ${ }^{1-3}$

Entre 1802 y 1805 aparece la primera edición de su libro Instituzioni Chirurgiche, finalizada la obra en cinco volúmenes, los más distinguidos médicos de Europa le dieron su más decidido apoyo. En esta pu- blicación encontramos nueva e invaluable información sobre importantes tópicos en cirugía de aneurismas, tumores, cáncer de útero y mama, estenosis uretral, una técnica propuesta para el drenaje de derrame pericárdico, tratamiento de las contusiones y heridas, los aparatos perfeccionados para el manejo de las fracturas y luxaciones y tratados completos sobre artritis, podagra, tétanos, mordeduras de serpientes, hidrofobia, angina de pecho, escrófulas (tuberculosis extrapulmonar ganglionar principalmente en el cuello). ${ }^{2}$

Después de una práctica de más de treinta años se imprime la última edición de su obra magna Instituzioni Chirurgiche, los primeros cinco volúmenes aparecen entre los años 1813 y 1814, antes de su muerte, y los tres últimos fueron editados entre los años 1815 y 1816 .

De interés particular para nuestra especialidad es la segunda parte, pp. 131-133. Sobre las lesiones violentas describe la fractura-luxación del antebrazo conocida hoy como lesión de Monteggia ${ }^{5,6}$ (Figura 2). Él escribió: «... At the end of the treatment the arm swelling has resolved, but only the dislocation

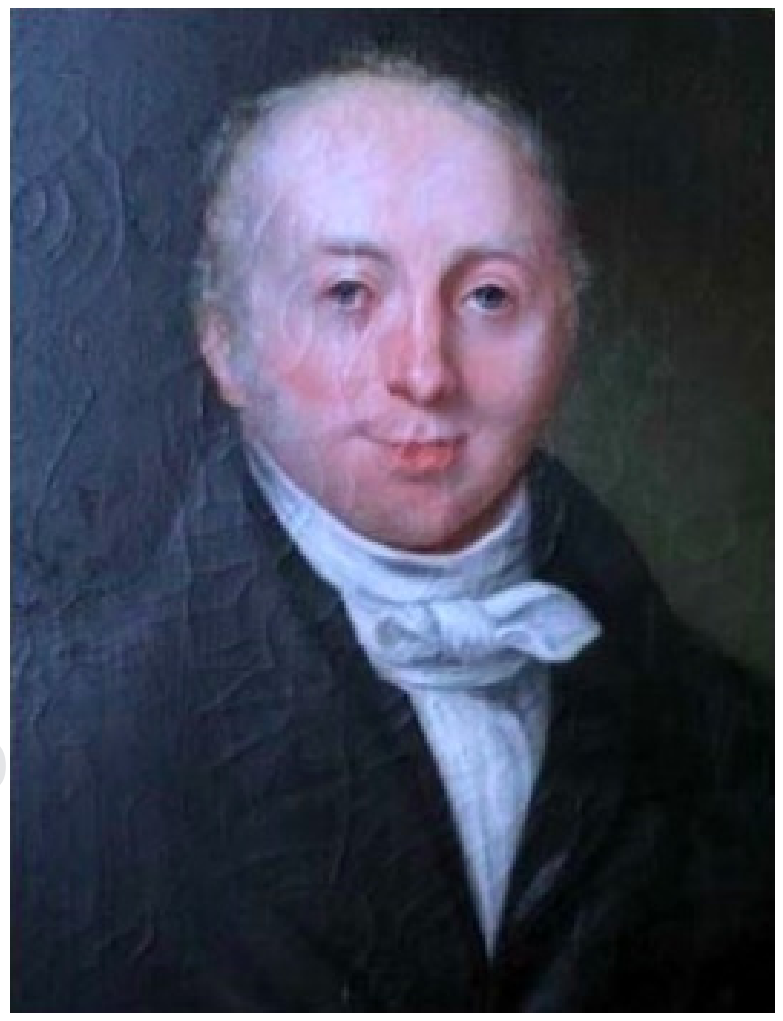

Figura 3: Abrahan Colles en su juventud (1773-1843). 
of the radius that was not easy to reduce.... I applied compression and a new bandage again to contain it, but it did not want to stay in place».5-7

El diagnóstico y ulterior manejo de las lesiones esqueléticas era a principios del siglo XIX sólo sobre bases clínicas, faltaban aún 81 años para el descubrimiento en 1895 de los Rayos X por Wilhelm Conrad Roentgen (1844-1923) de Wurzburgo, Alemania.

Es en 1814, segundo año de la última edición de Instituzioni Chirurgiche, donde G. B. Monteggia describe e ilustra precisamente esta patología.

Abraham Colles (1773-1843), también en 1814, publica sobre la fractura que lleva su nombre y el epónimo más célebre mundialmente conocido por la comunidad médica en general ${ }^{8,9}$ (Figura 3).

El 05 de enero de 1815, empleando el lenguaje de Enrico Acervi, «se siente muy indispuesto, parecía que sufría meramente un ataque febril con distensión abdominal, pero en pocos días le aparece erisipela cerca de la oreja derecha que gradualmente se disemina tomando un aspecto alarmante y que finalmente termina con su vida la noche del 17 de enero de 1815 ». $^{1,2}$
El término fractura-luxación de Monteggia fue acuñado en 1909 por el francés J. Perrin. ${ }^{6}$

\section{Referencias}

1. Acervi E. Della vita e degli studi di G:B: Monteggia. Della Stamperia Dova Milano 1816.

2. Acervi E. Biographical Memoir off Giambatista Monteggia, late Professor of Surgery at Milan. The Quarterly Journal of Foreign Medicine and Surgery, London, February, 1820.

3. Fusi F. Biografía di Giovanni Battista Monteggia Estratto della Rivista di Ortopedia e Traumatología, Vol. 24 N. 3, LuglioSettembre, Milano, 1956, 475-505.

4. Monteggia G.B. Annotazione pratiche sopra i male veneri. Giuseppe Galeazzi, Milano 1794.

5. Monteggia GB. Lussazioni delle ossa delle estremita superiori. In: Monteggia GB, editor. Instituzioni Chirurgiches. 2nd, Vol. 5. Maspero; Milan, Italy: 1814, 131-133.

6. Rehim SA, Maynard MA, Sebastin SJ, Chung KC. Monteggia fracture dislocations: a historical review. J Hand Surg Am. 2014; 39 (7): 1384-1394.

7. Rang M. The story of orthopaedics. 3rd ed. WB. Saunders Company, Philadelphia PA. 2000, 407-408.

8. Lee C. A trinity of eponymous irish surgeons. Abraham Colles (1773-1843) \& Colles Fracture of the Radius (1814), Volume 18, University of Pennsylvania Orthopaedic Journal. 2005-2006, 95-98.

9. Colles A. On the fracture of the carpal extremity of the radius. Edinb Med Surg J. 1814; 10 (38): 182-186. 